\title{
Diacronie
}

Studi di Storia Contemporanea

$N^{\circ} 8,4 \mid 2011$

Le sembianze di Clio: approcci alla storia

\section{Il Concilio Vaticano II nella stampa comunista italiana (1959-1965)}

\section{Lorenzo Ettorre}

\section{(QpenEdition \\ Journals}

\section{Edizione digitale}

URL: http://journals.openedition.org/diacronie/3504

DOI: $10.4000 /$ diacronie.3504

ISSN: 2038-0925

\section{Editore}

Association culturelle Diacronie

\section{Notizia bibliografica digitale}

Lorenzo Ettorre, « II Concilio Vaticano II nella stampa comunista italiana (1959-1965) », Diacronie [Online], Nº 8, 4 | 2011, documento 2, Messo online il 29 octobre 2011, consultato il 20 avril 2019. URL : http://journals.openedition.org/diacronie/3504; DOI : 10.4000/diacronie.3504 


\title{
Diacronie
}

2/

\section{Il Concilio Vaticano II nella stampa comunista italiana (1959-1965)}

\author{
Lorenzo ETTORRE*
}

Il pontificato di Giovanni XXIII e la convocazione del Concilio ecumenico Vaticano II rappresentarono svolte epocali, non solo per l'istituzione religiosa, ma anche per la società moderna Al fine di sviluppare lo spirito cattolico nella storia, questi eventi rinnovarono le modalità di intervento e le posizioni del cattolicesimo nell'età contemporanea. Attraverso una particolareggiata analisi della stampa comunista italiana, dove compaiono i giudizi di Togliatti, Donini, Spriano, le reazioni del Partito Comunista italiano rispetto al Concilio ne mostrano gli intenti, le aspettative e le strategie internazionali. Il risultato è un originale studio delle relazioni che intercorsero tra cattolici e comunisti e una visione generale della storia italiana di quel periodo.

\section{L'annuncio del Concilio}

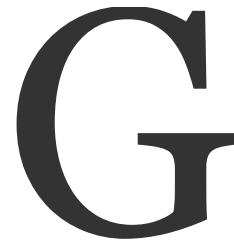

iovanni XXIII ha annunciato ieri la prossima convocazione del Concilio ecumenico al quale parteciperanno tutti i vescovi, i cardinali, ed altre gerarchie ecclesiastiche. Uno dei temi che si crede verranno discussi sarebbe quello della unità della Chiesa cattolica. L'ultimo concilio fu quello convocato da Pio IX nel 1869. Il prossimo dovrebbe riunirsi entro quest'anno, molto probabilmente a Roma ${ }^{1}$.

Queste le parole con cui «L'Unità», il 26 gennaio 1959, riporta la notizia dell'annuncio del XXI Concilio ecumenico della Chiesa cattolica, avvenuto il giorno precedente per opera di Giovanni XXIII.

\footnotetext{
${ }^{1}$ «Il Papa annuncia il 21 ${ }^{\circ}$ Concilio», in L'Unità, 26 gennaio 1959, p. 8.
} 
Nell'alveo complesso e articolato delle reazioni successive all'annuncio, particolare rilievo assumono i commenti giornalistici, sia nazionali che internazionali. Fu attraverso la stampa che la notizia della convocazione del Concilio riuscì in poco tempo a fare il giro del mondo, permettendo che tanti, sia tra i cattolici che tra i non cattolici, cogliessero nell'iniziativa di Giovanni XXIII un atto nuovissimo e gravido di significato $^{2}$. Non si trattava, come è ovvio, di giudizi chiari e definiti sul ruolo e l’incisività che il Concilio avrebbe avuto nella vita della Chiesa; nessuno, nemmeno il pontefice, era in grado di immaginare come e quanto questo avrebbe cambiato il volto del cattolicesimo3. Si trattava, piuttosto, della constatazione che l'annuncio di un Concilio ecumenico - il terzo in cinquecento anni - era di per sé un evento nuovo, un fatto eccezionale, per certi aspetti unico, di cui occorreva necessariamente prendere atto indipendentemente dal grado delle attese e delle speranze in esso riposte. Se queste furono le parole utilizzate da «L'Unità» per salutare l'annuncio, decisamente più singolare appare l'atteggiamento di «Rinascita», la nota rivista culturale del Partito Comunista italiano. In essa, durante tutto il 1959 e fino alla fine del 1960, non è presente alcun riferimento al Concilio. Il primo esplicito articolo comparirà soltanto nel novembre del 1960: due anni di assoluto e assordante silenzio. Il fatto appare ancora più eccezionale se si tiene in considerazione, da un lato, che «Rinascita» è un mensile di approfondimento culturale e sociale, e come tale manifesta interesse praticamente su tutte le grandi tematiche contemporanee - proprio per questo nelle sue pagine sono spesso presenti articoli sul mondo cattolico, verso il quale si è sempre dimostrata una considerevole attenzione - dall'altro, che altri organi di stampa in qualche modo legati al PCI affrontano direttamente tematiche connesse al Concilio. È il caso de «Il Calendario del Popolo», che nel numero del marzo 1959 ripercorre la storia dei Concili ecumenici della Chiesa offrendo al lettore delle nozioni basilari su cosa essi rappresentino e quali scopi perseguano ${ }^{4}$. Nell'assenza di ogni riferimento alla convocazione del Concilio da parte della più significativa rivista culturale del Partito, dunque, devono aver pesato alcuni importanti fattori. Tra questi, un ruolo centrale ebbe certamente la percezione delle profonde difficoltà nella sua effettiva, concreta,

\footnotetext{
2 Cfr ALBERIGO, Giuseppe, Il cattolicesimo verso una nuova stagione L'annuncio e la preparazione, in ID. (a cura di), Storia del concilio Vaticano II, vol. I, Bologna, Il Mulino, 1995, p. 49.

3 Non è un caso se quasi tutti i commenti successivi al discorso di S. Paolo restarono sul vago: non mancò chi tentò di improvvisare alcune previsioni circa temi e contenuti ma, nel complesso, la maggior parte degli organi di stampa si limitò a riportare la notizia, senza sbilanciarsi in approssimate e futuristiche supposizioni. Cfr ALBERIGO, Giuseppe, Breve storia del Concilio vaticano II (1959-1965), Bologna, Il Mulino, 2005.

4 DONINI, Ambrogio, «I Concili nella storia delle religioni», in Il Calendario del Popolo, X, 174, marzo 1959, p. 3281.
} 
realizzazione. Passare dalla "teoria" dell'annuncio alla "pratica" della convocazione doveva apparire un cammino tutt'altro che scontato: il silenzio imbarazzato dei cardinali presenti in S. Paolo al momento dell'annuncio, nonché le continue e reiterate pressioni della Curia, prima per fermare il Concilio poi per appropriarsene, contribuirono a ritenere poco probabile questo passaggio ${ }^{5}$. D'altronde, sfogliando le pagine della stampa comunista, non si può certo affermare che i vertici della Chiesa godessero di una qualche particolare fiducia. Al contrario, durante la fase preparatoria, la Santa Sede è ancora giudicata intrappolata tra due fuochi: la soffocante centralità della Curia romana - formatasi tutta durante la stagione degli anatemi e delle condanne dei decenni precedenti - e la pesante compromissione con il blocco occidentale-capitalista. Dalle stesse colonne de «Il Calendario del Popolo», per esempio, Ambrogio Donini ricorda che «negli ambienti più vicini al Vaticano si è parlato del Concilio come di una grossa operazione politica, destinata a dimostrare l'unità del mondo "occidentale" di fronte ai progressi compiuti in questi ultimi decenni dai popoli che si richiamano al socialismo ${ }^{6}$. Nulla nella sostanza sarebbe mutato nell'atteggiamento di Roma verso il mondo contemporaneo e, di conseguenza, nulla lasciava prevedere la benché minima possibilità di un aggiornamento e di un'apertura, come pure auspicato da Giovanni XXIII. Meno che mai attraverso un Concilio7. A

\footnotetext{
5 A tal proposito, val la pena ricordare che l'idea della convocazione di un Concilio ecumenico non era di per sé nuova. Già con i precedenti pontefici si era sotterraneamente fatto cenno a questa possibilità. All'inizio del suo pontificato, Pio XI aveva avuto l'idea di una ripresa del Concilio Vaticano I, il cui programma avrebbe dovuto comprendere l'elaborazione di una costituzione dogmatica e prese di posizione della Chiesa su socialismo e comunismo, Chiese orientali, Azione cattolica, scuola, ecc; l'idea era stata poi abbandonata perché - ha scritto Verucci - «la ripresa avrebbe fatto intendere che la Chiesa considerava superata la questione romana, che allora era invece tutt'altro che risolta sul piano formale». L'ipotesi di un proseguimento e completamento del Concilio del 1870 era stata anche di Pio XII, che nel 1948 aveva significativamente incaricato della sua preparazione il Sant'Uffizio, che elaborò progetti di risoluzione riguardanti le dottrine contrarie a quella della Chiesa, come il comunismo, e poi ancora la guerra, le missioni, l'Azione cattolica, il dogma dell'Assunzione della Vergine; l'idea fu ancora una volta abbandonata, sembra, questa volta, a causa dei contrasti che i progetti suscitavano. Cfr. VERUCCI, Guido, La Chiesa nella società contemporanea. Dal primo dopoguerra al Concilio Vaticano II, Roma-Bari, Laterza, 1999, p. 353. Il fallimento di queste iniziative contribuì verosimilmente ad avvolgere di pessimismo i giudizi di una parte della stampa comunista.

${ }^{6}$ DONINI, Ambrogio, I Concili nella storia delle religioni, cit.

7 La proclamazione del primato e dell'infallibilità personale del papa, unitamente alla pratica di governo della Chiesa, sempre più accentrata a Roma e nella Curia romana, «avevano notevolmente contribuito a privare di senso, a svuotare di contenuto, la partecipazione dei vescovi all'elaborazione dottrinale e alla prassi di governo, a far ritenere ormai tramontata l'epoca dei concili», Cfr. VERUCCI, Guido, La Chiesa nella società contemporanea, cit.
} 
dominare era ancora la percezione di una Chiesa fossilizzata nella difesa dello "status quo" e parte attiva del blocco occidentale in funzione anticomunista ${ }^{8}$.

Come è noto, fin dalla Svolta di Salerno, la linea politica del PCI era stata incentrata sul «tentativo di spostare e coinvolgere la Chiesa, la sua gerarchia e il suo clero in una posizione se non di diretto impegno democratico e antifascista, almeno di neutralità»9 . Gli anni compresi tra la fine della guerra e la morte di Pio XII, invece, non solo smentirono ogni possibilità di mutamento di rotta da parte della gerarchia ecclesiastica, ma testimoniarono con forza che a Roma, «la prospettiva di un ordine sociale cristiano ierocraticamente connotato, costituiva ancora [...] l'unica soluzione possibile ai giganteschi problemi che poneva al mondo la ricostruzione post-bellica ${ }^{10}$. A partire dai famosi radiomessaggi del 1944, infatti, era certamente la democrazia che il papa additava come via della ricostruzione, ma nella sua visione la democrazia, per essere veramente tale, non poteva che essere "cristiana". Sulla scorta di un retroterra culturale e ideologico siffatto, «riemergeva la tradizionale opposizione al comunismo, presentato come negatore di ogni libertà, diritto e valore e quindi radicalmente incompatibile con quella civiltà democratica e cristiana che avrebbe dovuto instaurarsi nel mondo per garantire pace e benessere ${ }^{11}$. Su queste basi politico-ideologiche, inoltre, si delineava la convergenza di posizioni tra Roma e le potenze occidentali, alimentatasi progressivamente nel tempo durante la "guerra fredda". Con la divisione del mondo tra blocco comunista - in cui si esercitava una reale oppressione verso le Chiese cristiane e blocco occidentale, egemonizzato dagli Stati Uniti - il cui cattolicesimo mostrava una crescente forza numerica ed economica - «Chiesa ed occidente trovarono un elemento comune nella decisa lotta contro il comunismo» ${ }^{12}$.

Non sorprende, dunque, che al novembre 1960, nel momento in cui si sostanzia l'attenzione di «Rinascita» verso il Vaticano II, i toni appaiono sferzanti e i giudizi, nel complesso, diffidenti. Dopo aver brevemente ricordato che nella lunghissima storia della Chiesa i Concili ecumenici non sono stati molti - ventuno in duemila anni - si fa riferimento a quello che viene identificato come il tratto distintivo comune: sebbene tutte queste assemblee abbiano rappresentato prese di posizione e svolte importanti nella politica generale della Chiesa, esse sono state «simili tutte in un solo aspetto, e

${ }^{8}$ Tra gli articoli che meglio illustrano un simile giudizio nei riguardi della maggior parte dei vertici ecclesiastici, cfr in particolare A.R., «Vescovi e laicismo», in Rinascita, maggio 1960, p. 336.

9 CASUlA, Carlo Felice, Cattolici-comunisti e sinistra cristiana (1938-1945), Bologna, Il Mulino, 1976, pp. 176-177.

${ }^{10}$ MENOZZI, Daniele, La Chiesa cattolica, in FILORAMO, Giovanni e MENOZZI, Daniele (a cura di), Storia del cristianesimo, l'età contemporanea, Roma-Bari, Laterza, 1997, p. 211.

${ }^{11}$ Ibidem.

12 Ibidem. 
cioè nel costante ritardo di decenni, a volte di secoli, con cui han seguito i mutamenti economici, sociali e politici che le avevano rese necessarie [...]. La "superiore saggezza politica della Chiesa" si è manifestata sempre dopo che la Chiesa aveva subito le conseguenze della propria intransigenza» ${ }^{13}$. Loris Gallico, autore dell'articolo, intravede nelle assemblee conciliari una sorta di argine, un "tampone" utilizzato per limitare le ferite inferte al mondo cattolico dalla sua stessa cronica intransigenza. I Concili non rappresenterebbero, né avrebbero mai rappresentato, un reale aggiornamento per il mondo cattolico; vengono visti, anzi, come l'estremo tentativo di difendere la dottrina, la gerarchia, e con esse l'ordine sociale ed economico costituito, dalle svolte della società che pure li avevano resi necessari. Proprio per questo le assemblee conciliari quali articolati strumenti delle forze della reazione - avrebbero sempre seguito e mai anticipato o coadiuvato i grandi mutamenti socio-economici dell'umanità. Non mancano esempi: da Ildebrando di Soana, «che riorganizza la Chiesa dopo che si era consumato lo scisma orientale», al Concilio di Trento, «che interviene dopo che la Riforma aveva sottratto al dominio del Vaticano buona parte dell'Europa», passando per l'ultimo Concilio, il Vaticano I, «tenuto dopo il fallimento dell'alleanza tra Trono e Altare». In definitiva, "più che a riconoscere i processi evolutivi in atto, i Concili han mirato in generale a frenarli» ${ }^{14}$. Questo interessato e persistente ritardo con cui la Chiesa avrebbe sempre affrontato le grandi svolte della storia non si limiterebbe alle assemblee ecumeniche del passato ma riguarderebbe anche il Vaticano II:

Il II Concilio Vaticano - scrive Gallico - è indetto quando una svolta decisiva si è compiuta nella storia. La crisi generale del capitalismo precipita; il socialismo ha vinto in un terzo del mondo. La coscienza delle principali leggi, o almeno di alcuni nessi dello sviluppo sociale è oramai presente nel pensiero degli uomini, e si diffonde, più o meno chiara, oltre la cerchia dei marxisti e del movimento operaio. La perdita dell'influenza della Chiesa su gran parte delle classi lavoratrici, la caduta della prospettiva di conquistare i popoli dei paesi «arretrati» al seguito delle potenze coloniali, sono fatti ormai generalmente ammessi, e se ne riconoscono come cause immediate non ragioni di ordine soprannaturale, ma i connubi col capitalismo e l'imperialismo ${ }^{15}$.

Il radicale cambiamento in atto in seguito al quale è stato annunciato il Concilio Vaticano II viene identificato chiaramente con la crisi del cattolicesimo moderno, a sua

13 GALLICO, Loris, «La preparazione del XXI Concilio ecumenico», in Rinascita, novembre 1960, p. 897 e ss.

14 Ibidem.

15 Ibidem. 
volta determinata proprio dalla soffocante compromissione con il capitalismo e l'imperialismo, cioè con il blocco Atlantico. Un capitalismo e un imperialismo, per giunta, che se qualche decennio prima potevano sembrare potenzialmente vincenti, tali da garantire benefici all'azione pastorale e politica della Chiesa - lo stretto rapporto tra missionari e Stati europei nelle terre coloniali ne rappresenterebbe un esempio - ora appaiono invece sempre più agonizzanti e sopraffatti dal cammino, considerato inarrestabile, delle forze del socialismo. La Chiesa, dunque - secondo Gallico - sarebbe stata trascinata nel baratro della regressione dal capitalismo, e la convocazione del Concilio sarebbe arrivata tardi, quando ormai la compromissione cattolica con l'Occidente era giunta a livelli tali da rendere impossibile ogni mutamento.

È un'interpretazione affascinante, tuttavia scarsamente utilizzabile nel caso del Vaticano II, verso il quale dimostra tutta la sua sostanziale esiguità. Pagando il tributo ad una visione filtrata col lumicino dell'ideologia, si fatica infatti a cogliere quanto di nuovo e originale stava accadendo, o era già accaduto, dopo l'annuncio del Concilio. Sebbene all'interno di maldestri e reiterati tentativi conservatori di impossessarsene, si erano già verificati alcuni avvenimenti di carattere indubbiamente innovatore, in modo speciale perché legati direttamente al pontefice e per questo non scontati. Anzitutto, il 14 luglio 1959, Giovanni XXIII aveva comunicato ufficialmente il nome del Concilio: si chiamerà Vaticano II. Il papa affermò così inequivocabilmente che sarebbe stato un Concilio nuovo e non un completamento del Vaticano I: «il Concilio, dunque, in quanto nuovo - ha rilevato Alberigo - ha un'agenda del tutto libera e aperta. Non sarà la continuazione di un'assemblea nata e poi sospesa in un contesto storico di conflitto e di pessimismo (il papa «prigioniero» nel Vaticano) ma costituirà una pagina bianca nella storia plurisecolare dei concili» ${ }^{16}$. In secondo luogo, nella fase ante-preparatoria preposta alla scelta dei temi da inviare alle future commissioni, il papa fece invitare ciascun vescovo ad indicare i problemi e gli argomenti che il Concilio avrebbe dovuto affrontare. Nei mesi successivi arrivarono in Vaticano circa duemila pareri (vota) da tutto il mondo; particolarmente significativo è il fatto che «la maggioranza di questi scritti testimoniava la sorpresa e il disorientamento: Roma non ordinava, ma chiedeva suggerimenti!»17. Il pontefice appariva in tal modo complessivamente meno conservatore dell'assemblea, cosa del tutto inconsueta dal momento che, di norma, si era sempre verificato il contrario. Il tutto, nel silenzio generale dei maggiori cronisti. In questo stesso scritto, che riveste un'importanza non trascurabile ai fini della nostra indagine, per la prima volta si fa riferimento ad uno dei temi che si presume saranno

\footnotetext{
${ }^{16}$ ALBERIGO, Giuseppe, Breve storia del Concilio vaticano II, cit., p. 29.

17 Ibidem, p. 28.
} 
trattati nella futura assemblea, e cioè l'unità tra le Chiese cristiane. Sebbene non passi momento in cui non si lamenti la segretezza e il riserbo in cui è ammantata questa fase preparatoria, anche «Rinascita», seguendo le notizie che trapelavano più o meno pubblicamente dagli ambienti vaticani, ipotizza che proprio l'unità dei cristiani sarà uno dei temi più significativi:

In questa situazione di crisi, e con questa direzione oltranzista, si è annunciato il Concilio. Il suo ordine del giorno è ancora segreto; alcune dichiarazioni del Papa sono state però rese di pubblica ragione: anzitutto una nota ufficiosa dell'Osservatore Romano ha rivelato che il Concilio «vuol essere altresì un invito alle comunità separate per la ricerca dell'unità»18.

$\mathrm{Al}$ di là delle motivazioni "ufficiali", non appare forse inutile indagare quali siano, secondo i commentatori comunisti, le reali motivazioni alla base dell'impegno verso l'unità e quali pensieri covassero in un simile innovativo approccio. Anche la risposta a tali quesiti non può prescindere dalla visione dell'universo cristiano e dalla percezione della Chiesa cattolica come di un'istituzione a "sovranità limitata", totalmente immersa nella contrapposizione della guerra fredda. Fermi restando fattori di carattere squisitamente religioso, l'annunciata svolta ecumenica è considerata derivante da altre spiegazioni, meno "trascendenti" e tali da riportarla entro coordinate più pragmatiche e "terrene":

Una considerazione più di ogni altra permette di ricondurre la svolta entro i suoi veri limiti. I cristiani di rito orientale, che non riconoscono l'autorità del Vaticano, sono valutati dalle fonti cattoliche a 165 milioni, di cui 15 milioni di monofisiti, meno di centomila nestoriani, e 150 milioni circa di greco-ortodossi. Di questi, 9 milioni di greci fanno capo al Patriarcato di Costantinopoli, alla Chiesa sinodale greca (78 diocesi) e alla Chiesa di Cipro, con una notevole diaspora nel Levante, in Europa, nelle Americhe. Ma la grande massa, decisiva, dei greco-ortodossi, per oltre il 90\%, sono slavi, dei Patriarcati di Mosca, di Bulgaria, di Jugoslavia e delle Chiese autocefale di Polonia e Cecoslovacchia; oppure romeni, georgiani, albanesi, ungheresi: tutti appartenenti a paesi del campo socialista. Ora, non appare ancora alcuno sforzo serio da parte del Vaticano, per un riavvicinamento con le Chiese ortodosse, senza confronti più importanti e più numerose, di questi paesi. Eppure esse hanno provato sempre la loro buona volontà ecumenica.19

${ }^{18}$ GALLICO, Loris, La preparazione del XXI Concilio ecumenico, cit.

19 Ibidem. Sulle colonne di «Vie Nuove» troviamo un giudizio non dissimile: «Come mai il concetto di unità con i fratelli separati resta pur sempre l'idea principe del Concilio? La risposta 
Si tende a sottolineare che l'indirizzo ecumenico di Giovanni XXIII non assume gli stessi contorni e le stesse caratteristiche verso tutte le confessioni cristiane. La Chiesa cattolica, cioè, non manifesterebbe la stessa disponibilità al dialogo verso l'intera cristianità ma svilupperebbe una sorta di graduatoria nell'attuazione pratica di questa svolta. Senza contare il chiaro e deciso impegno per il riavvicinamento con il mondo anglicano e protestante, questa diversità nell'approccio cattolico verso gli "altri” risalta all'interno della stessa cristianità di rito orientale: se, infatti, risoluto e determinato appare il fervore ecumenico verso il patriarcato di Costantinopoli, assai meno solerte appare nei confronti dei patriarcati di Mosca, Bulgaria, Cecoslovacchia ecc., di gran lunga più importanti rispetto al primo. Una difformità di approccio che ne svelerebbe il contenuto "politico":

L’insistenza vaticana nella infondata e oramai inefficace politica di discriminazione e di guerra fredda contro i paesi socialisti limita la portata e svela il contenuto politico di questi tentativi di riavvicinamento. Si punta su Costantinopoli, su Atene, e le più piccole Chiese autocefale, sul clero delle piccole frazioni ortodosse in America, sulle difficili condizioni amministrative in cui versa la Chiesa greca. (...). Nuoce alla Chiesa la mancanza di una politica autonoma, la sua compromissione con l'atlantismo e il colonialismo ${ }^{20}$.

La Chiesa non punterebbe affatto ad un riavvicinamento con le altre confessioni cristiane per motivi religiosi e pastorali ma, ancora una volta, utilizzerebbe tali finalità per rinsaldare e rafforzare il blocco occidentale contro quello socialista. L'azione di Roma si identifica come preminentemente politica. Tutto sarebbe in funzione della politica. La testimonianza di ciò è offerta dal caso della Cina, dove la Chiesa tra i cattolici che accettano il comunismo e Cian Kai-Scek sceglierebbe senza riserve quest'ultimo:

La durezza del Vaticano nei confronti della Cina è a prima vista incomprensibile; a differenza degli ortodossi e degli anglicani, i cinesi non rigettano il primato del

va ricercata nel quadro della politica internazionale e nell'estrinsecarsi dei rapporti di forze che sono alla base di questa politica. Indicendo questa grande assise di tutta la cattolicità (e non di tutta la cristianità) il Papato vuole allinearsi con tutto il peso della sua autorità al fianco di tutte le forze politiche, economiche, sociali e militari che il mondo cosiddetto occidentale ha schierato contro il mondo del socialismo». Cfr. PEZZELLA, Sosio, «Il Concilio: dicono unità ma vogliono ritorno all'ovile», in Vie Nuove, XVII, 4 gennaio 1962, p. 17.

${ }^{20}$ Ibidem. 
papa, non respingono alcun dogma, né alcuna forma di organizzazione: rifiutano solo di partecipare ad un'azione squisitamente politica, rivolta contro il potere popolare. E qui è la scelta: o la Cina e i suoi tre milioni di cattolici e i suoi 32 vescovi già eletti dal clero locale, o Cian Kai-Scek. Sinora il Vaticano ha scelto Cian Kai-Scek, così come, tra la Corea e Si Man Ri, aveva scelto Si Man Ri. Una svolta nei confronti della Cina non importerebbe decisioni di carattere teologico o «ecclesiologico», ma un diverso atteggiamento sulle questioni dei movimenti nazionali, del movimento operaio, del socialismo ${ }^{21}$.

La Chiesa continua ad essere giudicata un'istituzione filo-imperialista, un'organizzazione al servizio dell'Occidente arroccata nelle consuete sterili denunce di tutto quanto è moderno, e che non accetterebbe, per questo, la liberazione dei popoli colonizzati, cioè la loro inarrestabile marcia verso il socialismo. Ricreare anche soltanto "una parvenza di autonomia è certo uno degli scopi del Concilio, il quale dovrà necessariamente risolversi in un drammatico "confronto tra cristianesimo e mondo moderno»"22. Se il Concilio, secondo le aspirazioni giovannee, deve risolversi in un aggiornamento del cattolicesimo, esso non può prescindere dalla impellente necessità di separare le sorti della Chiesa da quelle del capitalismo. Proprio questo legame sostiene «Rinascita» - lungi dal cristianizzare la borghesia, ha determinato una pericolosa e dilagante secolarizzazione borghese all'interno del cattolicesimo, riducendo la Chiesa ad un mero strumento di lotta politica ${ }^{23}$.

Timidi e incerti segnali di apertura, compaiono a proposito della riproposizione di uno schema costante nella mentalità comunista, la distinzione tra la tradizionale chiusura della stragrande maggioranza dei vertici ecclesiastici e la forza potenzialmente progressista delle masse cattoliche. La prima tenacemente condannata per le proprie finalità politiche; le masse cattoliche valorizzate in quanto portatrici di una forza vitale e riformatrice. L'obiettivo è tener vivo e, se possibile, alimentare il rapporto con le masse popolari cattoliche, di cui viene sottolineata la sostanziale identità di interessi e vedute con quelle comuniste e socialiste:

\footnotetext{
${ }^{21}$ Ibidem.

22 Ibidem.

23 «Non appaiono ancora sintomi di rottura tra la Curia e i vari imperialismi. Gli elementi più reazionari della grande borghesia si sono nuovamente rivolti alla Chiesa, dopo il crollo dei fascismi, come all'estremo argine contro il socialismo, per incanalare e controllare quanto di realmente popolare affiora nel movimento cattolico. Ma non è la borghesia a «cristianizzarsi»: abbiamo invece la "secolarizzazione" della Chiesa che ha perduto sinanche la parvenza dell'autonomia politica. Ricreare questa parvenza è certo uno degli scopi del Concilio, il quale dovrà necessariamente risolversi in un drammatico "confronto tra cristianesimo e mondo moderno"». Cfr. ibidem.
} 
La sostanziale identità di interessi tra masse comuniste, socialiste e cattoliche si esprime nelle convergenze sempre più numerose e forti. E la spinta unitaria, in questi ultimi anni, è generale. È sempre più chiaro che all'universalità oggi non si può protendere persistendo a puntare sulle discriminazioni e sulle discordie, ma solo agendo per l'indipendenza e la fraternità delle nazioni, per la distensione internazionale, per il disarmo, per la pace. Questa è la linea nuova che si sovrappone alla vecchia, la linea di chi vuol sopravvivere e respingere lo spettro di una distruzione totale. Anche il Concilio non potrà non discuterne, sia pure nelle consuete forme mediate: perché è la linea che le masse cattoliche, sulla via della democrazia e del socialismo, si apprestano a seguire e seguiranno. ${ }^{24}$

Seppure all'interno di una visione sostanzialmente pessimistica, si attende che il Vaticano II, riformando la rigidissima struttura interna della Chiesa (che tende ad opprimere le forze vive del mondo cattolico), possa portare le masse cattoliche a sprigionare quell'energia e quello spirito progressista di cui sono strutturalmente portatrici. Proprio questa energia e questo spirito, convergendo con le aspirazioni comuniste e socialiste, è considerato il terreno d'incontro a partire dal quale ricostruire il dialogo dopo gli anni bui del centrismo e della guerra fredda. Si tratta di una divisione non certo nuova nell'universo comunista. Fin dalla sua fondazione, sebbene con fasi alterne, il tratto di fondo dell'approccio comunista verso il mondo cattolico è rappresentato da questa sostanziale differenziazione tra masse popolari e suoi rappresentanti ${ }^{25}$. La stessa strategia teorico-politica della democrazia progressiva che accompagnò il Partito nuovo di Togliatti fin dalla Svolta di Salerno ${ }^{26}$, si condensa in un certo senso proprio su analisi di questo tipo, volte a cogliere la complessità e l'importanza della presenza del cattolicesimo in Italia. Tra la fine degli anni Cinquanta e l'inizio degli anni Sessanta, tuttavia, con "l'evento Concilio" a fare da catalizzatore, la novità consiste nel riproporre questa distinzione tra masse cattoliche e gerarchia con una costanza progressiva, sostanziata da una fiducia crescente che da parecchio tempo appariva in crisi. Se è vero, infatti, che nel sostrato delle analisi storico-politiche del

24 Ibidem.

${ }^{25}$ Cfr. in proposito il fondamentale studio in due volumi di ZUNINO, Pier Giorgio, La questione cattolica nella sinistra italiana (1919-1939), Bologna, Il Mulino, 1975 e ID., La questione cattolica nella sinistra italiana (1940-1945), Bologna, Il Mulino,1977.

${ }^{26}$ Il leader del Partito Comunista italiano, prendendo atto dell'impossibilità di giungere al potere attraverso i consueti metodi rivoluzionari, aveva auspicato un graduale allargamento dell'influenza e dell'egemonia del suo partito fra le masse popolari italiane, indipendentemente dalla cultura e dalla tradizione di provenienza, per realizzare «una democrazia in progresso, in corso, in avanzata, che non restauri ma innovi». Su queste basi, «la democrazia progressiva rappresenta un regime fondato sulle larghe masse popolari e su una coalizione di forze democratiche». Cfr. SPRIANO, Paolo, Storia del Partito comunista italiano, La Resistenza. Togliatti e il partito nuovo, Torino, Einaudi, 1975, p. 390. 
PCI, pur nelle oggettive difficoltà degli anni del centrismo e della più acuta contrapposizione internazionale ${ }^{27}$, è sempre stata presente una politica della "mano tesa" verso i cattolici, come dimostrano alcune eloquenti prese di posizione del leader del $\mathrm{PCI}^{28}$, è pur vero che gli anni Cinquanta avevano contribuito a raffreddare il motore del dialogo e a renderlo più scopertamente sofferto.

\section{La convocazione dell'assemblea}

Nel tragitto che porta alla rivalutazione del Vaticano II, un punto di svolta è rappresentato dal 1962. Il Concilio, apertosi nell'ottobre, è uscito dall'astrazione e dalla vaghezza dell'annuncio per divenire una realtà. Ormai si potevano affrontare temi e argomenti a partire dallo spessore della discussione in aula piuttosto che dalla genericità delle ipotesi formulate. Proprio il passaggio dall'annuncio alla convocazione - inizialmente ritenuta per nulla scontata - rappresentò un notevole incentivo nell'attenzione comunista verso il Vaticano II $^{29}$. Già nella primavera di quell'anno, «Rinascita» lasciò trasparire alcuni segnali chiaramente orientati nel senso di una maggiore disponibilità. Un articolo del maggio 1962, per esempio, formula in modo del tutto innovativo il riconoscimento della effettiva ecumenicità dell'assemblea che "raccoglierà circa 2600 tra cardinali, vescovi, prelati, teologi, per la prima volta, d'ogni

\footnotetext{
${ }_{27}$ Per un'analisi complessiva e dettagliata della situazione sociale e politica dell'Italia negli anni Cinquanta, cfr fra gli altri CRAINZ, Guido, Storia del miracolo italiano. Culture, identità, trasformazioni fra anni cinquanta e sessanta, Roma, Donzelli, 2003; LANARO, Silvio, Storia dell'Italia repubblicana. L'economia, la politica, la cultura, la società dal dopoguerra agli anni '9o, Venezia, Marsilio, 1992; SCOPPOLA, Pietro, La repubblica dei partiti. Evoluzione e crisi di un sistema politico 1945-1996, Bologna, Il Mulino, 1997.

28 Nell'aprile del 1954, durante un discorso tenuto al Comitato centrale, in un clima internazionale molto teso, quasi a rischio di catastrofe atomica, a seguito della parziale sconfitta del centrismo dopo la "legge truffa", Togliatti ampliò le prospettive di avvicinamento chiamando in causa direttamente le masse cattoliche, e distinguendo tra questione cattolica e questione democristiana. Accennava così, diversamente da Rodano, alla possibilità di un dialogo che non passasse obbligatoriamente attraverso la dirigenza democristiana. Cfr. TOGLIATTI, Palmiro, Per un accordo tra il movimento comunista e il mondo cattolico allo scopo di salvare l'umanità dalla catastrofe di una guerra nucleare, discorso tenuto al Comitato centrale del Pci il 12 aprile 1954, in ID., Comunisti, socialisti, cattolici, Roma, Editori Riuniti, 1966, pp. 29-65. Questa apertura ai cattolici sul tema specifico del futuro dell'umanità, pur presentando possibili elementi di sviluppo originali e nazionali, era comunque in linea con la politica sovietica che aveva già denunciato il rischio di distruzione della civiltà mondiale. Cfr GOZZINI, Giovanni, $I l$ PCI nel sistema politico della Repubblica, in GUALTIERI, Roberto (a cura di), Il PCI nell'Italia repubblicana, Roma, Carocci, 2001, p. III.

29 Cfr. in particolare "L'eredità del "Vaticano I"», in Il Calendario del Popolo, XVIII, 216, ottobre 1962, pp. 5472-5473. Del tutto in linea con tale accresciuta attenzione è l'inchiesta sul Concilio e il mondo cattolico effettuata da Sosio Pezzella sulle pagine di «Vie Nuove», cfr. PEZZELLA, Sosio, «La crociata non si fa più», in Vie Nuove, XVII, 20 settembre 1962, pp. 5-11.
} 
razza, e di ogni continente"30. Se si considera che uno dei temi su cui si erano concentrate le accuse della stampa comunista nei riguardi della Chiesa era proprio la perdita del suo originario afflato "cattolico", appare chiara una svolta negli indirizzi e negli orientamenti della rivista. Cosa è accaduto di nuovo? Rispondere a questa domanda equivale a toccare un punto essenziale nella nostra questione. Sta lentamente ma inesorabilmente mutando il giudizio sul pontefice. In precedenza, la politica di Giovanni XXIII era stata sostanzialmente equiparata a quella del suo predecessore, senza variazioni di sorta sulle tradizionali accuse; ora, invece, emergono orientamenti e tendenze che rimarcano la novità rappresentata dalla sua elezione. Si comincia a prendere atto che con Giovanni XXIII la Chiesa poteva effettivamente intraprendere un percorso di revisione interna e, con questo, di apertura al mondo contemporaneo. A ciò contribuirono diversi importanti fattori: anzitutto, la stessa apertura del Concilio rappresentò l'occasione per riconoscere che il papa, rifuggendo ogni astratta retorica, era fermamente intenzionato a dare un seguito reale all'annuncio; era la prova che le intenzioni del pontefice non solo erano fondate ma anche decise. In secondo luogo, altri convincenti avvenimenti contribuirono a ristrutturare la visione comunista: i numerosi interventi di Giovanni XXIII per ribadire la necessità di un aggiornamento della Chiesa (che avrebbe dovuto procedere ad una riflessione su se stessa anziché disseminare nuove e severe condanne); il suo impegno costante per il raggiungimento della pace mondiale; le trattative, innovative e coraggiose, portate avanti col governo sovietico per permettere ai vescovi dell'Est di giungere a Roma. Questi ultimi due punti in particolare, favorirono in modo straordinario l'avvio della distensione internazionale tra i due blocchi, divenuto allora il principale caposaldo della politica estera sovietica ${ }^{31}$. Ancora nel maggio del 1962, dalle colonne di «Rinascita» si riconosce che soltanto un uomo come Roncalli avrebbe potuto percepire l'urgente necessità di un Concilio, sì da alimentare speranze ed attese: "pervenuto al Soglio attraverso una lunga esperienza pastorale e diplomatica in alcuni dei settori più tormentati del mondo cattolico è riuscito a trarre una visione realistica e complessiva delle condizioni della Chiesa romana" 32 .

La figura del pontefice, del resto, era l'unica in grado di trasmettere dei segnali in qualche modo rassicuranti. Nessuna apertura poteva provenire dall'assemblea,

3o PIERATOZZI, Libero, «Contrasti tra i cardinali sul Concilio Vaticano II», in Rinascita, 5 maggio 1962, p. 9.

${ }^{31}$ Cfr in particolare WERTH, Nicolas, Storia della Russia nel Novecento. Dall'Impero russo alla Comunità degli Stati indipendenti 1900-1999, Bologna, Il Mulino, 2000.

32 PIERANTOZZI, Libero, Contrasti tra $i$ cardinali sul Concilio Vaticano II, cit. Sempre in relazione alla crescita di stima nei confronti di Roncalli cfr "Giovanni XXIII», in Il Calendario del Popolo, XIX, 224, aprile-maggio 1963, p. 5831. 
dilaniata dai contrasti che opponevano i conservatori alle istanze dei progressisti. Agli osservatori comunisti non sfuggivano gli ostacoli frapposti all'aggiornamento dalla parte più retriva dell'assemblea, in particolare i vescovi italiani, spagnoli e americani; proprio per questo, le buone intenzioni mostrate da Giovanni XXIII venivano rappresentate come l'unico terreno su cui far fiorire speranzose ipotesi di cambiamento. Non a caso, cogliendo una delle caratteristiche più significative di questo primo periodo, «Rinascita» non perdeva occasione di evidenziare che l'assemblea convocata in S. Pietro era profondamente divisa non solo sulle singole questioni ma anche sui temi e gli argomenti più generali. Su queste divergenze, significativo il confronto effettuato da Libero Pierantozzi tra l'intervento dell'arcivescovo di Genova, card. Siri, che in merito al Concilio ammonisce che «aggiornamento non significa mutazione di principi»33, e quello dell'arcivescovo di Colonia, card. Frings, il quale azzarda invece che «uno dei compiti più reali dei quali il Concilio dovrà occuparsi sarà di aprire la Chiesa, ancor più di quanto non sia stato fatto finora, a quella grande molteplicità dello spirito umano che le spetta in quanto cattolica»34.

Un'analisi complessiva, benché sommaria, della situazione politica e sociale nazionale, alcune considerazioni generali sulla situazione internazionale e sulla “distensione" fra i blocchi, nonché una visione d’insieme della strategia interna del PCI e del suo inscindibile legame con Mosca, aggiungono dei tasselli decisivi nella ricostruzione del mosaico che fa da sfondo alla rivalutazione comunista di Giovanni XXIII e del "suo" Concilio. Unitamente alle motivazioni finora evidenziate, infatti, il progressivo avvicinamento a papa Roncalli e al Concilio affonda le sue origini in un complessivo rinnovamento delle prospettive del Partito Comunista italiano verso la Chiesa cattolica. Già con il IX Congresso del gennaio 1960, il PCI riconosceva l'importanza del ruolo della Chiesa e del movimento cattolico per orientare una considerevole parte della classe operaia, del mondo contadino, e dei ceti medi ${ }^{35}$. Nelle tesi del X Congresso del dicembre 1962 - in concomitanza con la chiusura della prima sessione conciliare e a pochi mesi dal fondamentale intervento di papa Roncalli nella crisi cubana - il PCI svolse «il primo passo effettivo verso l'apertura ai cattolici»36, affermando che l'aspirazione ad una società socialista non poteva non «trovare nella coscienza religiosa uno stimolo di fronte ai drammatici problemi del mondo

\footnotetext{
33 PIERANTOZZI, Contrasti tra i cardinali sul Concilio Vaticano II, cit.

34 Ibidem.

35 Cfr. Per una nuova maggioranza, dal rapporto al IX Congresso del PCI, Roma 30 gennaio - 4 febbraio 1960, in TOGLIATTI, Palmiro, Comunisti, cit. pp. 212-216.

${ }^{36}$ SCIRÉ, Giambattista, La democrazia alla prova. Cattolici e laici nell'Italia repubblicana degli anni Cinquanta e Sessanta, Roma, Carocci, 2005, p. 126.
} 
contemporaneo»37. Si giunse, poi, a quello che a buon diritto può «essere considerato l'approdo più maturo della riflessione togliattiana sulla questione religiosa: il discorso di Bergamo, in cui il leader comunista optava per un reciproco riconoscimento di valori cristiani e comunisti, facendo un grande passo avanti in direzione dell'affermazione di valori quali la laicità e il pluralismo nella società $3^{8}$. Un simile progressivo strutturarsi dell'analisi comunista della questione cattolica trovava nuova linfa, e a sua volta se ne alimentava, dai rivoluzionari segnali di apertura lanciati a più riprese da Giovanni XXIII, di cui si è detto. Non va dimenticato, inoltre, che gli inizi degli anni Sessanta rappresentarono l'apice della distensione internazionale e dell'affermazione di una duratura pace mondiale. Proprio il tema della pace rappresentò un catalizzatore sensazionale nell'avvicinamento comunista al "nuovo corso" intrapreso dalla Chiesa. Esso, infatti, se da un lato è sempre stato presente nell'orizzonte culturale e intellettuale del PCI - come dimostra l'impegno del Partito per il movimento dei Partigiani della Pace - in questo momento assume un'importanza per così dire "strategica", in quanto del tutto in linea con gli orientamenti e le strategie della politica estera sovietica39. Un simile contesto contribuiva a rendere proprio il pontefice l'interlocutore privilegiato della politica comunista.

Le parole con cui Ambrogio Donini saluta l'avvio dei lavori dell'assemblea lasciano spazio a poche interpretazioni. Sebbene all'interno delle consuete cautele, il mutamento di rotta è ormai avvenuto. E così, dopo aver celebrato non senza retorica il valore dei comunisti e dei lavoratori italiani, i soli che «hanno nel sangue il senso dell'«ecumenicità», del valore universale della solidarietà e della fratellanza (...), in una società minacciata dalla torbida morale del profitto borghese e dal ricatto atomico dei suoi difensori»40, l'intellettuale piemontese non può nascondere il forte interesse del suo Partito «per il raccogliersi a Roma, da tutte le parti del mondo, di centinaia e

\footnotetext{
37 X Congresso del PCI (Roma, 2-8 dicembre 1962), Roma, Editori Riuniti, 1962, p. 668. ${ }^{8}$ SCIRE, Giambattista, La democrazia alla prova, cit. Secondo Scirè, l'evento conciliare fu guardato con crescente interesse dai comunisti italiani anche a seguito della pubblicazione del volume di Gozzini intitolato "Concilio aperto" (GOZZINI, Mario, Concilio aperto: con una scelta di testi del Magistero Ecclesiastico, Firenze, Vallecchi, 1962). Afferma Scirè: "Il volume fu il primo passo effettivo e concreto, da parte di alcuni laici, in direzione di un'azione attiva nel miglioramento del rapporto tra Chiesa e società civile». Cfr. Ibidem, pp. 131-132.

39 Esaminando più da vicino le risoluzioni del X Congresso del PCI, «Critica Marxista» (rivista bimestrale fondata nel gennaio del 1963 da Luigi Longo e Alessandro Natta) analizza l'intervento di Togliatti incentrato sulla necessità di giungere al più presto ad una nuova fase nei rapporti USA-URSS. Per il segretario comunista, "parlare oggi di possibilità di pacifica coesistenza è poco. Di fronte alle prospettive catastrofiche di un conflitto atomico, si deve affermare che la pacifica coesistenza è, non solo per gli Stati socialisti ma per quelli capitalistici e per tutta l'umanità, una necessità inderogabile”. Cfr ALICATA, Mario, "Coesistenza e lotta socialista», in Critica marxista, I, gen-feb 1963.

$4^{\circ}$ DONINI, Ambrogio, «Il Concilio», in L’Unità, 11 ottobre 1962, p. 1.
} 
centinaia di esponenti della gerarchia cattolica, per un lavoro che potrebbe rappresentare, per la sua stessa iniziale impostazione e per la gravità dell'ora, qualcosa di nuovo, qualcosa di diverso dal vecchio spirito di crociata ideologica e di condanna politica e sociale" ${ }^{41}$. Si tratta di una breccia notevole, tanto nella forma quanto nel contenuto. Ancor più emblematica appare l'analisi del discorso di apertura del pontefice, Gaudet mater ecclesia. In esso viene ravvisato il definitivo abbandono delle tradizionali posizioni della Chiesa, quelle stesse che l'avrebbero condotta ad intossicarsi di "occidentalismo" e che avevano fatto dell'anticomunismo un'ossessione tanto ardita quanto controproducente. In questo discorso si vedevano condensate gran parte delle aspettative comuniste, tanto che ciò che fino a poco prima appariva impossibile semplicemente a dirsi, sembrava ora compiersi, inverarsi, esprimersi, quantomeno dal punto di vista dell'annuncio programmatico. L'allocuzione giovannea venne accolta come una novità dirompente, soprattutto se paragonata al clima che si respirava un secolo prima, durante la convocazione dell'ultimo Concilio ecumenico:

L'allocuzione pronunciata dal pontefice Giovanni XXIII ha confermato la novità di questo Concilio del ventesimo secolo, novità del resto già implicita nella composizione intercontinentale dell'assemblea e nei temi proposti alla discussione. La novità balza agli occhi, sol che si pensi che un secolo fa il Concilio indetto da Pio IX si apriva sotto il segno del «Sillabo» e della lotta frontale al mondo moderno e al pensiero moderno. [...] Mentre l'allocuzione del Papa ha affermato e sollecitato ieri, al di là di ogni dubbio, un orientamento opposto, un positivo e generale «aggiornamento» dinanzi alla realtà e ai problemi del nostro tempo. E non solo di un aggiornamento si tratta ma di una rettifica, dell'abbandono anzi di tutta una linea che, fin sotto l'ultimo pontefice, compromise la Chiesa in una identificazione piena col mondo occidentale e le sue storture: mentre oggi - secondo l'espressione di Giovanni XXIII - la Chiesa intende sentirsi e anzi si sente «finalmente libera da tanti ostacoli di natura profana» ${ }^{2}$.

Spriano parla, dunque, di "rettifica" delle posizioni della Chiesa di fronte alla complessità dei problemi del mondo moderno. Una rettifica resa ancor più corposa dall'assenza totale di anatemi e condanne - secondo uno spirito di accoglienza dei bisogni, e anche degli errori, del mondo moderno - che per la sua carica imponente e per il suo afflato missionario non ha eguali nella storia del cattolicesimo:

Se fossero sorti dubbi sul metodo e sull'indirizzo generale da adottare in questo compito apostolico (quello del Concilio, nda), Giovanni XXIII ha ulteriormente precisato che «al giorno di oggi la Chiesa preferisce far uso della medicina della

\footnotetext{
41 Ibidem.

42 SPRIANO, Paolo, «Il discorso del papa», in L'Unità, 12 ottobre 1962, p. 3.
} 
misericordia piuttosto che della severità»; in altri termini, piuttosto che con la condanna si intende operare con la persuasione e con il dialogo. Non è chi non veda come una simile impostazione suoni una sorta di archiviazione per quelle scomuniche e per quel clima di crociata e di intolleranza che avevano caratterizzato il pontificato precedente 43 .

Secondo il consueto atteggiamento, tuttavia, anche questa rilevante apertura viene circoscritta in ambiti e limiti che la imprigionano in una rete di diffidenze mai del tutto sopite. Viene sottolineato che si tratta pur sempre di premesse, di annunci, che come tali non hanno ancora nulla di concreto e definito e che anzi incontrano degli ostacoli notevoli nella realizzazione 44 . Tuttavia, ciò non toglie nulla della svolta che si sta consumando, come testimonia il giudizio sull'appello lanciato dai vescovi al mondo intero, pochi giorni dopo l'avvio dei lavori. Il messaggio, dedicato all'autopresentazione della Chiesa e della sua missione, era teso a mostrare agli uomini la solidarietà dei cattolici sui grandi problemi contemporanei. Era chiaramente un messaggio rivolto all'intera umanità, in particolare su due aspetti, la pace e la giustizia sociale. Come ha notato un vescovo africano, "non stavamo più al chiuso, nelle nostre piccole capanne" 45 . Questa presa di posizione dei vescovi, innovativa e straordinaria nella sua grandezza, unitamente al discorso di apertura del papa, rappresentano l'apice delle aspettative nei confronti del Concilio. Mai prima d'ora era stato, e mai in seguito sarà presente, un giudizio tanto appassionato e carico di attese come quello che traspare evidentissimo dalle colonne de «L'Unità»:

Il documento, che riveste un'eccezionale importanza proprio perché è il primo nella storia a godere dell'appoggio dell'episcopato mondiale, ha un contenuto formalmente religioso, ma nella sostanza conferma il «nuovo corso» della Chiesa cattolica e la «politica rinnovatrice» iniziata da Giovanni XXIII sin dai primi giorni del suo pontificato. Non contiene condanne né anatemi né esclusioni o

\footnotetext{
43 Ibidem. Sosio Pezzella, attento e costante osservatore del Concilio per conto di «Vie Nuove», cogliendo nell'accettazione del mondo moderno un tratto distintivo dell'opera giovannea, non esita ad affermare: «Il passo del discorso che ha più colpito è stato il diffuso senso di ottimismo con cui è stata presentata la nostra epoca. Contro i "profeti di sventure», che sono poi coloro i quali sognano sempre la Chiesa religiosamente "armata", in lotta contro il socialismo e il laicismo, il Papa ha invece sottolineato le favorevoli congiunture in cui attualmente il cattolicesimo vive nel mondo». Cfr PEZZELLA, Sosio, «Il ventunesimo», in Vie Nuove, XVII, 18 ottobre 1962, p. 5 .

44 Cfr. SPRIANO, Paolo, Il discorso del papa, cit.

45 ALBERIGO, Giuseppe, Breve storia del Concilio vaticano II, cit., p. 47.
} 
discriminazioni. È un appello alla giustizia sociale e alla pace: e si conclude con una velata ma ferma condanna degli strumenti di distruzione atomica. 46

Il primo periodo conciliare, così, viene considerato come una svolta per l'assemblea in quanto determinò una limitazione marcata della propensione centralizzatrice della Curia, garantendo notevoli libertà all'episcopato mondiale. La lenta e contrastante continuazione dei lavori determinò l'affermazione di un clima sempre più caratterizzato dal dispiegarsi di aperture e innovazioni, che proprio come tali vengono recepite dagli osservatori comunisti.

Lo schema Ottaviani, relativo alle Fonti della Rivelazione, è un altro esempio di come lentamente arrivi a prevalere una visione decisamente più ottimista verso il Vaticano II. Lo schema, sostenendo che sia la Scrittura che la Tradizione avessero pari dignità come Fonti della Rivelazione, rischiava di ostacolare sul nascere il dialogo con le altre confessioni cristiane che così timidamente si era avviato proprio grazie al Concilio. Nel periodo immediatamente successivo all'annuncio, come abbiamo visto, il mondo comunista bolla il perseguimento dell'unità tra i cristiani come un bieco tentativo per compattare il blocco occidentale in funzione antidemocratica. Proprio per questi presunti scopi politici filo-occidentali veniva fermamente condannato. In questo periodo, invece, a Concilio iniziato e con la discussione nel vivo del suo sviluppo, il timore che si manifesta leggendo le pagine comuniste è esattamente l'opposto, e cioè che la ricerca dell'unità venga interrotta. Spriano sottolinea con malcelata soddisfazione la frattura che va approfondendosi fra i padri conciliari, crescendo il numero di coloro che si oppongono ad Ottaviani, «tanto da rendere lecita l'ipotesi che (...) si sviluppi una delle battaglie di principio più forti e laceranti nella Chiesa»47. Pur sottolineando che «naturalmente è fuori di luogo anche solo pensare che la corrente del Concilio oppostasi così aspramente allo schema Ottaviani, si muova con criteri di revisione simili a quelli dei protestanti», cioè rivendicando la dignità di Fonti della Rivelazione soltanto alle Scritture, indagate con i moderni criteri scientifici, «essa però rivendica, sulla base dello studio più fresco delle discipline bibliche, una interpretazione che si dimostri più conciliante, e che tenta di distinguere il senso "autentico" religioso di alcune asserzioni della Bibbia dalle altre affermazioni che dovrebbero invece essere considerate come documenti puramente umani, frutto degli antichi modi di narrare e di dire»48. Viene così sostenuto l'aggiornamento dei metodi di

46 MAGAGNINI, Franco, «Il Concilio fa sua l'impostazione di Giovanni XXIII», in L'Unità, 21 ottobre 1962, p. 1.

47 SPRIANO, Paolo, «Guerra fredda contro Ottaviani», in L'Unità, 17 novembre 1962, p. 3.

48 Ivi. 
ricerca storica e scientifica, tali per cui si giunga a realizzare un terreno di incontro e di dialogo con i protestanti, in luogo delle reciproche accuse che contrasterebbero irreversibilmente i rapporti con gli altri cristiani. Su «Rinascita» viene confermata la tendenza comunista a sostenere e approfondire il dialogo ecumenico, prendendo posizione contro ogni ipotesi che in qualche modo possa rischiare di metterlo a repentaglio:

se la sostanza del testo approntato dal capo del Sant'Uffizio dovesse essere confermata non si comprenderebbe l'ulteriore permanenza in San Pietro dei rappresentanti delle comunità religiose protestanti e ortodosse (...). Se tale testo venisse approvato avrebbe ribadito e forse irreparabilmente approfondito uno dei solchi più profondi che divide il mondo cristiano.49

Tralasciando l'analisi comunista nel merito dello schema, ciò che val la pena rilevare è questo netto cambiamento circa il perseguimento dell'unità - prima condannato, ora auspicato - dovuto essenzialmente a due motivazioni. Da un lato, all'interno dello scontro ideologico tra progressisti e conservatori, tutto ciò che in qualche modo arrivi a contrastare le tendenze e l'operato di quest'ultimi è bene accetto nell'ottica comunista: in tal senso, contrariando le posizioni del capo del S. Uffizio, card. Ottaviani, la reazione dell'ala progressista non può che essere giudicata positivamente. Dall'altro lato, e in modo decisamente più significativo, il mutamento nel giudizio sull'ecumenismo appare rilevante perché esso, da strumento di lotta anti-socialista, diviene fattore in grado di contribuire al dialogo e alla distensione, un contributo essenziale al rafforzamento della pace nel mondo. In quanto caposaldo della politica estera sovietica, la ricerca di un nuovo ordine nei rapporti Est-Ovest diveniva al contempo uno degli interessi peculiari del PCI. Anche in questo caso, e forse in modo ancora più nitido, tutto ciò è sostanzialmente spiegabile con la costante e crescente fiducia nei confronti di Giovanni XXIII.

Tutto il primo periodo è un crescendo impetuoso e inarrestabile della stima e del credito nei confronti di papa Roncalli. Non è assolutamente casuale che proprio ora si intensifichino articoli che riportano, spesso con viva preoccupazione, l'evoluzione dello stato di salute del pontefice, che in quel momento iniziano drammaticamente a regredire. Dopo una serie di articoli della fine del mese di novembre in cui si constata un peggioramento delle sue condizioni di salute, il primo dicembre del 1962, in prima

49 PIERANTOZZI, Libero, Lo Schema Ottaviani, in «Rinascita», 24 novembre 1962, p. 2. 
pagina si riporta la notizia che «il papa migliora»50. Il tono e il contenuto dell'articolo lasciano intendere un vero e proprio senso di liberazione per l'avvenuto miglioramento: è sempre più evidente come le accresciute speranze nei confronti del Concilio siano strettamente connesse alla figura dirompente di Roncalli.

\section{Il concilio di Paolo VI}

Il 1963 è un anno fondamentale. Nel settembre è prevista la riapertura dei lavori: in riferimento alla lunga pausa della prima intersessione e ai mai sopiti tentativi della Curia di impossessarsi del Concilio, si attende di capire se l'assemblea abbia interiorizzato gli orientamenti di Giovanni XXIII oppure se, al contrario, essendosi sciolta e in un certo senso "dispersa" per lungo tempo, abbia lasciato cadere quanto di nuovo c'era stato durante il primo periodo. Direttamente legato a questi interrogativi, il 1963 è anche l'anno della morte di Giovanni XXIII e dell'ascesa al soglio pontificio del cardinale di Milano, G. B. Montini. Vi era un'attesa enorme di conoscere la volontà di Paolo VI circa il proseguimento dell'opera di Roncalli e, una volta appurata, di conoscere quali fossero i suoi orientamenti.

Il trapasso di Giovanni XXIII rappresentò un evento drammaticamente straordinario. Tutti furono costretti a farci i conti, indipendentemente dalla cultura e dalla tradizione di provenienza. I toni utilizzati da «Rinascita» furono caratterizzati da accenti chiaramente celebrativi, che se non giunsero mai ad una retorica vaga e sentimentale non furono neppure alieni da una compartecipazione commossa e sincera. Giovanni XXIII viene descritto come un pontefice che "ha operato per ridar spazio alla sua Chiesa nel mondo moderno, ponendosi in modo nuovo dinanzi agli essenziali problemi del nostro tempo" ${ }^{51}$. Un pontefice che partendo dalla positiva rivalutazione dell'uomo, delle sue esigenze e delle sue aspirazioni, è riuscito nell'intento di unificare ciò che fino a qualche tempo prima sembrava destinato a restare diviso per sempre, e così "uomini semplici d'ogni parte, atei e credenti, si sono trovati in questi giorni accumunati nel profondo e spontaneo cordoglio per la sua scomparsa" ${ }^{52}$. Abbandonando la forza apparente delle condanne per abbracciare la forza reale dell'accoglienza, ha contrastato le posizioni più austere e retrive del cattolicesimo giungendo a dare "l'altolà al segretario del S. Uffizio, in vena di nuovi anatemi (verso i

\footnotetext{
50 «Il papa migliora», in L'Unità, 1 dicembre 1962, p. 1.

${ }^{1}$ PIERANTOZZI, Libero, «L'eredità di Giovanni XXIII», in Rinascita, 8 giugno 1963, p. 3.

${ }^{2}$ Ibidem.
} 
comunisti, nda), dicendo con fermezza: «lasciateli stare, sono anch'essi figli miei»"53. Il decesso di Roncalli fu anche una circostanza privilegiata per tracciare un bilancio del Concilio fino a quel momento. Proprio a seguito di quanto detto finora, il giudizio è tutt'altro che negativo: la prima sessione è definita letteralmente «una fase silenziosa ma non meno importante»54. Un modo per dire che, benché non sia stato approvato alcun testo - e, anzi, poco lavoro è stato effettivamente svolto - ciò non significa che non si siano ottenuti risultati "concreti", essendosi smosse le acque di una Chiesa da secoli ripiegata su astratti arroccamenti e lasciando entrare, pur attraverso limiti e contraddizioni, effettive ipotesi di cambiamento.

Il 1963, tuttavia, segna un'altra fondamentale svolta: dopo l'apice del consenso e dell'apprezzamento, inizia un periodo di timori e inquietudini legati, come detto, alla figura del nuovo pontefice. Questi saranno talmente profondi da trascinarsi, con alti e bassi, lungo tutta la durata del Vaticano II. Benché Paolo VI si fosse immediatamente affrettato a comunicare che il Concilio sarebbe continuato, non mancarono voci, anche autorevoli, che cominciarono a nutrire seri dubbi circa le modalità e gli approcci della sua azione pastorale. In questo senso, nessun giudizio sarebbe stato certamente più autorevole di quello dello stesso Togliatti, che per la prima volta dall'inizio del Concilio affronta su «Rinascita» tematiche legate al mondo cattolico. Secondo il leader comunista, è impossibile conoscere i nuovi indirizzi che prenderà la Chiesa in questo momento di passaggio; si possono elaborare soltanto congetture, per quanto significative. Per questo motivo, tutto quanto viene scritto dagli organi di stampa deve essere visto con cautela e diffidenza, perché altro non è che «l'espressione più o meno esplicita dei desideri e persino delle richieste che le classi dirigenti borghesi formulano nei confronti della Chiesa. $\mathrm{E}$ la base di questi desideri e di queste richieste è sempre la dottrina che fa della religione lo strumento di potere (instrumentum regni) di chi sta alla sommità della scala economica e sociale»55. Il segretario del Partito sembra voler mettere in guardia da facili ottimismi e semplicistiche previsioni, ricordando che $\mathrm{i}$ tentativi della classe borghese di utilizzare a propri usi e consumi la Chiesa, soprattutto con il nuovo pontefice, sono sempre presenti e in agguato. In merito al Concilio, ai suoi occhi appare con certezza che la convocazione sia stata una necessità inderogabile, dovuta alla crisi nella quale era precipitato il cattolicesimo a causa degli orientamenti di Pio XII. Sulle sue spalle, infatti, «gravava il peso non soltanto della politica estera atlantica, ma delle dittature fasciste di Portogallo e Spagna, del regime coloniale in

\footnotetext{
53 Ibidem.

54 PIERANTOZZI, Libero, «Il destino del Concilio», in Rinascita, 1 giugno 1963, p. 6.

55 TOGLIATTI, Palmiro, «Da Giovanni a Paolo», in Rinascita, 29 giugno 1963, p. 1.
} 
sfacelo, dell'autoritarismo francese e del militarismo tedesco» ${ }^{6}$. Secondo Togliatti, non occorreva certo "grande acutezza politica per avvertire che queste circostanze sono all'origine sia delle correnti manifestatasi e rafforzatesi negli ultimi anni in seno alle gerarchie, sia della stessa azione svolta da Giovanni XXIII»57. Diffidenze e preoccupazioni Togliatti mette in risalto anche nel finale dell'articolo, nel quale si domanda come mai la persona di Giovanni XXIII sia stata circondata da tanto entusiasmo. Egli protesta contro coloro i quali giudicano questo affetto legato esclusivamente alla pacatezza del suo temperamento. Afferma in proposito: "è chiaro che non basta, per spiegarselo, rievocare l'aspetto del pontefice scomparso, la sua capacità di contatto e comprensione col popolo, l'assenza di quei tratti che rendevano quasi impossibile una simpatia di massa per Pio XII" ${ }^{5}$. Per il leader comunista una simile giustificazione appare insufficiente, elaborata da chi vuole limitare le aperture giovannee alla sua sola persona, ad un suo modo di essere e fare, tralasciando volutamente la portata mondiale che invece recano con sé. È convinto che le masse popolari, vere motrici della storia, siano state convinte da ben altro:

\begin{abstract}
Due sentimenti hanno dominato, secondo noi, nell'animo delle masse popolari, alla morte del pontefice, determinando il particolare carattere che assunsero in tutto il mondo, ma specialmente in Italia, le così ampie manifestazioni di compianto $\mathrm{e}$ cordoglio. Una fu la gioia di aver visto il Capo della Chiesa perorare la causa della pace, non più soltanto con le tradizionali espressioni dei sacerdoti, un po' vuote di senso immediato, ma con le parole di un politico impegnato in una battaglia contro posizioni da smascherare e avversari da battere. L'altra fu la speranza di veder chiusa l'epoca dei fanatismi e finito veramente il tempo delle crociate.59
\end{abstract}

Giudicare positivamente l'azione di Giovanni XXIII equivale non soltanto a tessere le lodi di un personaggio assolutamente fuori dagli schemi, nuovo nelle forme e nei contenuti del suo agire, ma delinea anche il sentiero sul quale si dovranno condensare i rapporti futuri tra i cattolici e i comunisti. Togliatti, nell'ammonire quanti riducono la portata dell'opera giovannea al carattere bonario della sua indole, rilevava un rischio per nulla secondario: perdere, insieme al pontefice, anche le sue riforme, trasformandole in un sentimentalismo vago intriso di rimpianti. Egli vuole, invece, separare il pontefice dalla sua azione, Giovanni XXIII dalle sue intuizioni, dando a queste ultime una sorta di "vita a sé stante", una ragion d'essere incarnata nella sua

\footnotetext{
${ }^{56}$ Ibidem.

57 Ibidem.

$5^{8}$ Ibidem.

59 Ibidem.
} 
persona ma ad essa superiore. Era questo un modo, forse l'unico, per dire al mondo che non dissipare quanto di buono e grande si era verificato dal 1958 al 1963 non significava attendere l'arrivo di un "nuovo Roncalli", di un uomo cioè che avesse le sue stesse caratteristiche caratteriali ed umane - ipotesi quanto mai astratta e fumosa - ma equivaleva ad una presa di coscienza dell'«oggetto effettivo» della sua azione, dell'importanza straordinaria che essa ha rappresentato per gli uomini di ogni religione e continente. Secondo Togliatti, tale «oggetto effettivo» non poteva che essere la lotta per la pace e il raggiungimento della giustizia sociale, cioè le aspirazioni più autentiche e originali che riteneva albergassero nel cuore di ogni uomo. Roncalli fu davvero grande non perché dotato di una "superiore saggezza", destinata a scomparire con la sua persona, ma perché ha saputo guardare alla radice le attese più intime dell'umanità. Proprio per questo, al nuovo pontefice Togliatti non chiedeva un'imitazione della bontà di Roncalli, ma di riconoscere come un fatto ineludibile e irriducibile la centralità assoluta che il suo insegnamento, le sue aperture, la sua azione hanno rappresentato nel mondo, e con ciò assecondarle. Il leader del PCI ben sapeva che gli uomini, anche i più grandi, passano, e difficilmente rimangono "esempio" se mal si è compreso l'originario contenuto delle loro gesta. Togliendo ogni velo di una superficialità inevitabilmente passeggera, il Segretario del PCI invitava così a puntare dritto al cuore del lascito giovanneo, a "prenderlo in mano", a farci i conti, sì da renderlo effettivamente un punto di non ritorno. In altre parole, voleva che fosse il nuovo pontefice a piegarsi alla riforma di Giovanni e non il contrario: chiedeva un ruolo preminente dell'oggetto anziché del soggetto, delle innovazioni anziché delle interpretazioni. D’altronde, secondo la visione di Ercoli, era proprio su questo oggettivo comune retroterra che poteva sostanziarsi l'auspicata alleanza tra masse comuniste e cattoliche, che la convocazione del Concilio aveva senza dubbio riproposto fra i temi all'ordine del giorno nel PCI. L'intervento del leader comunista, inoltre, appariva una vera e propria apologia del comunismo, dei suoi ideali e delle sue prospettive, teso come era a mostrare il ritardo col quale il vertice della Chiesa giungeva a posizioni che il PCI da tempo professava.

Tuttavia, i primi provvedimenti di Paolo VI - attento a far valere ovunque il peso delle proprie decisioni - sembrano prendere una direzione assai diversa da quella auspicata da Togliatti, a tal punto che appare impossibile non rilevare alcuni irrigidimenti nell'atteggiamento comunista verso la riapertura dei lavori della seconda sessione. In particolare, notevole scetticismo suscitò il discorso di Paolo VI alla Curia romana, nel quale il pontefice annunciò il proposito di una sua maggiore presenza in assemblea come arbitro indiscusso delle contese tra conservatori e innovatori. In un 
certo senso, si tratterà di una maggiore centralizzazione rispetto alla sostanziale libertà respiratasi durante la prima sessione:

dopo aver rievocati lo stupore e le apprensioni Curiali affiorati all'improvviso annuncio della convocazione del concilio (papa Giovanni, nella sua schiettezza, parlò di resistenza), nel corso di una specialissima udienza, aveva freddamente prescritto una gioconda sintonia professando certezza che, d'ora innanzi, mai più sarebbero trapelate esitazioni e disparità di giudizio in ordine ai valori del pontefice. 60

Vengono in tal modo evidenziate le due linee programmatiche dell'azione politica e pastorale del pontefice: ribadire il primato papale, soprattutto nei confronti dei vescovi, e «raggiungere un'unanimità di vedute e una convergenza al centro tra le posizioni della maggioranza e della minoranza conciliare ${ }^{61}$. In nome dell'unanimità di vedute secondo «Rinascita» - il papa starebbe tentando di sottomettere l'assemblea al proprio supremo volere. Il suo atteggiamento inizia per questo ad essere considerato da più parti come sostanzialmente ambiguo. Né può passare inosservato che, contrariamente all'atteggiamento di Roncalli, Montini fissa direttamente i punti essenziali all'ordine del giorno della nuova sessione. Si tratta: “1) della conoscenza, della coscienza della Chiesa; 2) della sua riforma; 3) della «ricomposizione di tutti i cristiani nell'unità»; 4) del colloquio della Chiesa col mondo contemporaneo"62. Giovanni XXIII aveva volontariamente lasciato all'assemblea la possibilità di fissare l'agenda e i contenuti dei lavori, limitandosi a tracciarne le linee guida. Montini, invece, non esita ad elencare chiaramente le sue personali direttive in merito all'imminente ripresa dei lavori. Il quotidiano comunista afferma perciò che "l'elencazione di questi scopi quanto il tono e le espressioni usate dal pontefice nell'illustrarli consentono già di cogliere alcune caratteristiche nuove nell'orientamento della Chiesa”. Non solo. Se durante la prima sessione Giovanni XXIII aveva parlato con toni commossi ed entusiasti di fronte al mondo contemporaneo, lasciando intendere l'avvio di un nuovissimo atteggiamento della Chiesa - Paolo VI pone "come problema essenziale di questa seconda sessione conciliare la volontà di riformare la struttura della Chiesa”. Giovanni XXIII avrebbe guardato all'esterno, Paolo VI all'interno:

6o PIERANTOZZI, Libero, «Addomesticheranno lo Spirito santo?», in Rinascita, 28 settembre 1963, p. 14.

61 SPRIANO, Paolo, «Prospettive diverse al Concilio indicate da Paolo VI», in L'Unità, 30 settembre 1963, p. 1 .

62 Ibidem. 
Basterebbe, in proposito, confrontare l'allocuzione con cui Giovanni XXIII apriva l'11 ottobre dell'anno scorso il Concilio per vedere uno spostamento dell'asse stesso dei lavori. Là l'accento era messo sul rapporto della Chiesa col mondo contemporaneo, sulla sua vocazione pastorale e sullo slancio ottimistico verso «un nuovo ordine dei rapporti umani»; qua, mutando la prospettiva dei lavori, l'esame introspettivo sulla natura della Chiesa, sui suoi problemi, sul suo rinnovamento, viene considerato come preliminare e determinante e all'ottimismo fa seguito un grave pessimismo. ${ }^{63}$

D'altro canto, lo schema De Ecclesia - relativo proprio alla struttura interna della Chiesa - è oggetto di notevole interesse da parte comunista. In particolare il capitolo III, riguardante il rapporto tra il papa e i vescovi, sarà costantemente presente nelle pagine e negli articoli dei commentatori comunisti. Nel merito specifico del dibattito si prende espressamente posizione a favore delle tesi della collegialità, difendendo diritti e prerogative episcopali rispetto al centralismo pontificio. L'origine di questo atteggiamento non va ricercata, come è ovvio, in motivazioni di carattere "religioso", che ben poco interesse suscitavano nella pubblicistica del PCI. Sono le ripercussioni "politiche" nel dialogo con il mondo contemporaneo a costituire la ragione del favore verso la collegialità. L'introduzione nella Chiesa di elementi di democraticità è visto come fattore che eviti la concentrazione di poteri in una sola persona, il papa; rischio, questo, che l'ascesa di Paolo VI ha fatto tornare prepotentemente alla ribalta ${ }^{64}$. Garantire maggiore autonomia agli episcopati nazionali significava scongiurare l'ipotesi che il cattolicesimo tornasse ad essere uno strumento di lotta nelle mani dell'Occidente, nonché permettere una più estesa possibilità di incontro fra le diocesi e le varie realtà politiche locali, favorendo il dialogo con il mondo moderno. E così, proprio dall'interno del capitolo III dello schema De Ecclesia, non sorprende l'attenta disamina di uno speciale documento, il Motu proprio "Pastorale munus", attraverso il quale il papa intende concedere ai vescovi più ampi privilegi. Esso viene aspramente condannato non soltanto per il suo modesto e contraddittorio contenuto ma anche per le modalità contingenti con le quali si è giunti ad approvarlo. Il documento, secondo «L'Unità», rappresenta un esempio lampante di come le feroci controversie tra maggioranza e

63 Ibidem. Cfr. anche PEZZELLA, Sosio, «Sua Santità il presidente», in Vie Nuove, XVIII, 3 ottobre 1963, pp. 5-9. In tale occasione Pezzella, dopo aver espresso giudizi sostanzialmente in linea con quelli riportati, non esita ad evidenziare che se a livello teorico le posizioni sul mondo contemporaneo tendono a coincidere, "quando si giunge all'atteggiamento pratico che la Chiesa deve assumere verso di esso, quale profonda differenza si nota tra i due Pontefici!».

${ }^{64}$ Cfr PEZZELLA, Sosio, «L'ombra di Paolo VI sul Concilio», in Vie Nuove, XVIII, 5 dicembre 1963, p. 9 . 
minoranza in Concilio abbiano incentivato la tendenza accentratrice del pontefice, esautorando di fatto l'assemblea di una buona fetta di potere decisionale ${ }^{65}$.

L'ancora alla quale restano legate le attese di un cambiamento della Chiesa cattolica restano i fermenti nuovi che la convocazione e l'apertura del Concilio hanno fatto definitivamente emergere. Senza nascondere timori e pericoli, sono proprio questi fermenti a lasciare aperte ragionevoli speranze sul futuro e a far ritenere improbabile, se non del tutto impossibile, un ritorno della Chiesa allo status quo ante. Il segnale confortante viene individuato dalla presenza in assemblea di personaggi di rilievo che hanno manifestato la convinzione di proseguire l'opera e il cammino di Giovanni XXIII. Fra tutti, il card. Agostino Bea. Quantunque si sottolinei quanto arduo sia trarre un bilancio del Concilio - perché “il «Vaticano II» resta più che mai aperto: tutti gli schemi nodali sulla «rivelazione», sulla struttura ecclesiale, sull'ecumenismo rimangono sul tappeto"66 - la presenza in assemblea di uomini come il card. Bea rappresenta la concreta possibilità di caute ma sostanziali speranze di innovazione ${ }^{67}$.

Alla fine del settembre del 1964 il capitolo sulla collegialità appare definitivamente superato. Il prevedibile scontro fra le varie correnti in Concilio non c'è stato, tutto è stato tacitamente "messo a posto" dal solito compromesso68. Ciò che appare di innovativo nella valutazione della votazione finale su questo capitolo appare il riferimento, ancora blando per la verità, all'importanza che assumerà il periodo del post-concilio. Preso atto della presenza di alcune timide innovazioni sulla collegialità, si comincia a riflettere sull'importanza non soltanto dei testi redatti dal Concilio quanto delle loro future applicazioni:

\section{Il principio della collegialità è affermato (o riaffermato) e pertanto l'episcopato non è fatto più di semplici delegati pontifici. Tuttavia l'esplicazione di tale collegialità può avvenire solo «con e sotto» il Papa. Tutto qui. In futuro da questo seme - per}

\footnotetext{
${ }^{6}$ Cfr SAVIOLI, Arminio, «Oggi il Papa concede più potere ai vescovi», in L'Unità, 3 dicembre 1963, p. 2., e SAVIOLI, Arminio, «Marginali concessioni di Paolo VI ai vescovi», in L'Unità, 4 dicembre 1963, p. 2. Sul carattere generale dell'azione pastorale di Paolo VI cfr. PEZZELLA, Sosio, «Montini: retromarcia a S. Pietro», in Vie Nuove, XIX, 26 marzo 1964, p. 17. Nel lamentare che il modello di Montini sembra essere sempre più la "conquista ideologica" di Pio XII piuttosto che l'apertura al mondo di Giovanni XXIII, Pezzella mette in luce il contenuto eminentemente politico del suo operato: «La cura particolare che la Chiesa sta dedicando affinché ovunque si senta e si avverta la sua presenza - afferma tra l'altro - sembra rivolta a bilanciare proprio la presenza del PSI al governo».

66 PIERANTOZZI, Libero, «Agostino Bea, gesuita moderno. Un personaggio chiave del Concilio Vaticano II», in Rinascita, 30 novembre 1963, p. 3.

${ }_{67} \mathrm{Ci}$ si riferisce, in particolare, all'atteggiamento tenuto dal card. Bea nelle discussioni sullo schema riguardante gli ebrei e la libertà religiosa, dove il porporato tedesco ha mostrato uno spiccato afflato ecumenico. Cfr. Ibidem.

${ }^{68}$ Cfr. GRILLO, Giorgio, «Potere dei vescovi: prevale la linea del compromesso», in L'Unità, 23 settembre 1964, p. 3 .
} 
dirla in termini consoni all'argomento - potranno nascere nuovi e interessanti germogli; ma la coltivazione è ancora tutta da fare. Del resto il tempo non è misura delle questioni della Chiesa cattolica. ${ }^{69}$

Val la pena sottolineare, tuttavia, il rilievo attribuito a queste pur limitate aperture sulla collegialità, soprattutto in paragone al clima di austera chiusura dei decenni precedenti. Viene valorizzato, in particolare, come la destra in Concilio abbia ricevuto un duro colpo dall'approvazione di questo capitolo. L'accettazione di alcune prerogative episcopali - si afferma - «è un fatto, un avvenimento, che ha scosso le coscienze di molti e limitato il potere dei pochi; non è certamente uno schema avanzato, progressista nel senso proprio e assoluto del termine, ma è sufficiente ad assestare alla Curia un duro colpo, togliendole molti degli indiscussi privilegi di cui godeva con Pio $\mathrm{XII} \gg 70$.

Nel frattempo, i lavori in assemblea continuano e con essi proseguono le analisi dei commentatori comunisti su alcune fra le più importanti questioni dibattute, prima fra tutte la dichiarazione sulla libertà religiosa. Suscitando notevoli scontri tra conservatori e vescovi provenienti dai paesi a "religione mista", la dichiarazione - afferma Pierantozzi - ha indubbiamente «intaccato, a cento anni esatti dalla promulgazione dell'enciclica di Pio IX Quanta cura e del Sillabo, un impianto dottrinale tradizionale fondato sulla affermazione intollerante ed esclusiva della verità ecclesiale cattolica; da qui era fatta derivare la liceità del ricorso al potere civile per contrastare, e reprimere, le pratiche manifestazioni della libertà di culto e di coscienza»71. Quantunque consapevole delle fessure aperte dalla dichiarazione sulla libertà religiosa nel rigido dogmatismo cattolico, Pierantozzi sostiene che l'abbandono di queste cosiddette trincee della tradizionale intolleranza cattolica, pone delle domande brucianti sul loro reale significato:

Tale revisione ha origine soltanto da una più moderna e serena valutazione del pluralismo religioso e culturale oggi affermato nel mondo? È soltanto frutto di una volontà ecumenica che il «Vaticano II» ha ora il compito di rendere esplicita mediante atti che dovrebbero, almeno, appannare il ricordo di tragiche persecuzioni e guerre di religione? ${ }^{22}$.

\footnotetext{
69 GRILLO, Giorgio, «Ottaviani attacca il testo sulla libertà religiosa», in L'Unità, 24 settembre 1964, p. 3.

${ }^{70}$ GRILLO, Giorgio, «Collegialità: definitiva sconfitta della "destra” », in L'Unità, 1 ottobre 1964, p. 2.

${ }_{71}$ PIERANTOZZI, Libero, «Post-Concilio indiano», in Rinascita, 7 novembre 1964, p. 15.

72 Ibidem.
} 
Certamente questi motivi sono presenti, né potrebbe essere altrimenti, «ma a noi sembra che quello essenziale possa ricercarsi in un calcolo globale, letteralmente cattolico, cioè universale, dei rapporti di forza, diciamo così, religiosi non più circoscritti - come cento anni or sono - nell'ambito di un'Europa predominante, impegnata nella politica di assoggettamento o di egemonizzazione degli altri continenti»73. In altre parole, così come per il testo sulla collegialità, anche nel caso della libertà religiosa, l'origine prevalente dell'azione della Chiesa sarebbe un calcolo strumentale finalizzato ad estendere la sua egemonia nell'Europa e nel mondo. Quando l'Europa era dominante rispetto agli altri continenti, fino alla Seconda Guerra Mondiale, e il cattolicesimo prevaleva indiscusso in Europa, non vi era motivo alcuno per garantire un diritto quale quello della libertà religiosa; anzi, con gli Stati coloniali che dominavano praticamente nel mondo, garantirlo avrebbe potuto significare allontanare da Roma individui o popoli che ne erano da tempo legati. Ora, invece, con un cattolicesimo costretto ai margini dai vari progressi cui è andata incontro l'umanità, l'unico modo per lasciare aperte possibilità di nuove "conquiste" cattoliche è proprio quello della difesa della libertà religiosa. Il fine è esattamente lo stesso, ciò che cambia è il contesto storico e culturale 74 .

Come si vede, si alternano senza sosta posizioni di condanna ad improvvise e repentine aperture. Sul finire della terza sessione, tuttavia, è tempo di primi, definitivi, bilanci. Il Concilio non appare più quello innovativo e impetuoso che fu durante la prima sessione, l'attuale pontefice ha poco a che vedere con la "rivoluzionaria" personalità di Giovanni XXIII, le energiche attese suscitate dall'uno e dall'altro stanno inesorabilmente lasciando spazio a giudizi distaccati e formali. Ma un elemento non si può in alcun modo trascurare: il Concilio ha scosso la Chiesa, la sua struttura interna e il suo ruolo nel mondo. Le istanze nuove che premono sulla Chiesa «sono molteplici e consistenti»75. Esse «vengono dal mondo moderno, dal seno stesso del cattolicesimo attraverso pastori e fedeli lontani da Roma che per tanta parte vivono in tale mondo moderno - e da rappresentanti della gerarchia ecclesiastica»76. A rafforzare un simile intendimento hanno senza dubbio contribuito i recenti avvenimenti in assemblea: in particolare, le vivacissime discussioni in aula e la sensazionale sconfitta della destra di Ottaviani che, con ogni mezzo, aveva tentato di affossare il capitolo sulla collegialità.

\footnotetext{
73 Ibidem.

74 Cfr. ibidem.

75 GRILLO, Giorgio, «Cauto ammodernamento per l'apostolato laico», in L'Unità, 7 ottobre 1964, p. 2.

76 Ibidem.
} 
Con la conclusione della terza sessione è avvenuta una conferma, per nulla scontata, di impressioni e ipotesi che già erano state esplicitate in passato. In ambito comunista, ci si è scontrati con l'evidenza che dal 1958 in avanti si sono avuti dei segnali oggettivamente orientati in senso innovatore, i quali, non possono né devono essere sottovalutati, soprattutto in un momento come quello della famosa "settimana nera", ennesima riprova dell'esistenza di tenaci manovre dell'opposizione che avrebbero potuto prevalere. Un nuvolo di grigie possibilità ancora aleggia impetuoso sul futuro del Concilio.

L'analisi della quarta ed ultima sessione appare confermare questa visione d'insieme. Dalle colonne di «Rinascita», il solito Pierantozzi si chiede: «ritorno allo status quo ante, dunque? Giovanni XXIII è ora soltanto una canna agitata dal vento nel deserto del gerarchismo dogmatico, una voce solitaria che si dilegua nel frastuono di un clericalismo inviperito dall’incubo di quegli anni?»77. Non può non rispondersi che «dire soltanto ciò sarebbe fare certamente un consuntivo superficiale ed erroneo » ${ }^{8}$. Stesso orientamento emerge dall'analisi finale sullo schema XIII, relativo espressamente al rapporto tra la Chiesa e il mondo contemporaneo. Un paragone serrato tra le numerose stesure cui è incorso lo schema è inevitabile, soprattutto in un momento in cui «l'ennesima relazione ha rivelato i definitivi mutamenti imposti (non certo in senso migliorativo) al testo»79. Vengono così esaminati i punti ritenuti più significativi. Per quanto riguarda il capitolo sull'ateismo, «la richiesta di una condanna "espressa e formale" del comunismo è stata respinta»; tuttavia, «nel paragrafo relativo appare ora una nota a margine della quale vengono richiamate la precedente condanna e le encicliche di Pio XI, Pio XII, Giovanni XXIII e Paolo VI»80. Arretramenti vengono invece ravvisati per quanto concerne il capitolo sulla guerra - altro esempio della tendenza al compromesso - il cui testo riporta in auge il delicato concetto di "guerra giusta" che con la Pacem in terris sembrava definitivamente superato. Anche riguardo il capitolo su matrimonio e famiglia, a dominare è la stessa impressione di trovarsi dinanzi un testo smussato delle parti più estreme: il vecchio classico «fine primario» dell'unione coniugale, la procreazione dei figli, viene riaffermato «senza che le altre

\footnotetext{
77 PIERANTOZZI, Libero, «Contro Concilio Vaticano - Si tenta di demolire l'eredità di Giovanni XXIII», in Rinascita, 1 maggio 1965, p. 5.

78 Ibidem.

${ }^{79}$ GRILLO, Giorgio, «Concilio: no alla condanna del comunismo», in L'Unità, 3 dicembre 1965, p. 2.

80 Ibidem.
} 
finalità siano trascurate» ${ }^{81}$. Nel complesso, appare evidente che il Concilio abbia "deciso di non decidere", lasciando che tutto fosse rimandato a disposizioni future. Eppure, anche nel merito dello schema XIII non mancarono segnali di apertura, talvolta notevoli. Fra tutti, degni di attenzione sono la notizia che «le scomuniche che si scambiarono reciprocamente nel 1054 la Chiesa cattolica romana e la Chiesa ortodossa di Costantinopoli sono state annullate per decisione comune delle due Chiese medesime» ${ }^{82}$, e la fine dello Statuto della Congregazione del S. Uffizio, eretta da Paolo III nel 1512, che "per quattrocento anni è stato lo strumento centrale e assolutista da usare contro ogni eresia (reale o ritenuta tale)»83. Quello che nei secoli era divenuto il centro dell'integralismo più intransigente cambia nome, compiti, e in qualche misura, strutturazione. Per «L'Unità» è inevitabile sostenere che «una certa aria nuova entra anche nel fosco palazzo del cardinale Ottaviani» 84 .

Il Vaticano II si chiude solennemente l'8 dicembre 1965. Agli osservatori, comunisti e non, è chiaro che quanto si è mosso con esso deve essere seguito, valorizzato, sostenuto da un fondamentale impegno futuro nell'applicazione delle risoluzioni. Dello straordinario spirito giovanneo molto si è perso per strada durante gli anni, molto altro si è tenuto in piedi a fatica, altro ancora si è sostanzialmente modificato, non sempre in meglio. È chiaro, tuttavia, che da ogni parte si avverte la lucida percezione che la Chiesa del 1965 non sia la stessa del 1959. Gli schemi e le dichiarazioni conciliari, tutte perfettibili, laddove pedissequamente e tenacemente seguiti, potrebbero dare l'abbrivio a cambiamenti strutturali e finanche epocali. Così come, di contro, potrebbero essere affossati e congelati come una bella ma inconsistente parentesi nella vita della Chiesa. Non può esservi nessun giudizio netto poiché tutte le strade sono ancora aperte, tanto da far dire a Ferrara: «il Concilio è finito. Il Concilio comincia»85. Unica certezza appare la speranza che lo spirito del Concilio venga «trasferito alla "storia" 86.

\footnotetext{
81 Ibidem. Cfr. anche CHIARANTE, Giuseppe, «Famiglie fuorilegge», in Vie Nuove, XX, dicembre 1965. In particolare: «Su questi temi ha finito per prevalere in Concilio il richiamo alla prudenza, ma la discussione apertasi nell'aula di S. Pietro non è certo passata invano».

82 GRILLO, Giorgio, «Annullate le scomuniche fra cattolici e ortodossi», in L'Unità, 6 dicembre 1965, p. 5 .

83 GRILLO, Giorgio, «Riforma del S. Uffizio, «si» allo schema XIII, Giubileo straordinario», in L'Unità, 7 dicembre 1965, p. 3.

84 Ibidem.

85 FERRARA, Maurizio, «Il Concilio e la storia», in L'Unità, 10 dicembre 1965, p. 1.

86 Ibidem.
} 


\section{* L'autore}

Laureato in Storia presso l'Università Tor Vergata di Roma con una tesi sul Partito comunista italiano, ora è dottorando presso l'Università della Tuscia di Viterbo. Dopo aver approfondito alcune tematiche relative ad aspetti specifici del colonialismo europeo in Africa, il suo ambito di interesse attuale è incentrato sull'analisi dei rapporti tra "mondo cattolico" e "mondo comunista" nell'Italia repubblicana.

URL: < http://studistorici.com/progett/autori/\#Ettorre >

\section{Per citare questo articolo:}

ETTORRE, Lorenzo, «ll Concilio Vaticano II nella stampa comunista italiana (1959-1965)», Diacronie. Studi di Storia Contemporanea: Le sembianze di Clio: approcci alla storia, 29/10/2011,

URL:< http://www.studistorici.com/2011/10/29/ettorre_numero_8/ >

Diacronie Studi di Storia Contemporanea $\beta$ www.diacronie.it

Risorsa digitale indipendente a carattere storiografico. Uscita trimestrale. redazione.diacronie@hotmail.it

Comitato di redazione: Marco Abram - Giampaolo Amodei - Jacopo Bassi - Luca Bufarale - Alessandro Cattunar - Alice De Rensis Barbara Galimberti - Deborah Paci - Fausto Pietrancosta - Martina Sanna - Matteo Tomasoni - Luca Zuccolo

Diritti: gli articoli di Diacronie. Studi di Storia Contemporanea sono pubblicati sotto licenza Creative Commons 2.5 Possono essere riprodotti a patto di non modificarne i contenuti e di non usarli per fini commerciali. La citazione di estratti è comunque sempre autorizzata, nei limiti previsti dalla legge. 